\title{
Influences of hydration force and elastic strain energy on the stability of solid film in a very thin solid-on-liquid structure
}

\author{
C. Y. Yang and Ya-Pu Zhao a) \\ State Key Laboratory of Nonlinear Mechanics (LNM), Institute of Mechanics, Chinese Academy of Sciences, \\ Beijing 100080, China
}

(Received 25 August 2003; accepted 19 December 2003)

\begin{abstract}
Since hydration forces become very strong at short range and are particularly important for determining the magnitude of the adhesion between two surfaces or interaction energy, the influences of the hydration force and elastic strain energy due to hydration-induced layering of liquid molecules close to a solid film surface on the stability of a solid film in a solid-on-liquid (SOL) nanostructure are studied in this paper. The liquid of this thin SOL structure is a kind of water solution. Since the surface forces play an important role in the structure, the total free energy change of SOL structures consists of the changes in the bulk elastic energy within the solid film, the surface energy at the solid-liquid interface and the solid-air interface, and highly nonlinear volumetric component associated with interfacial forces. The critical wavelength of one-dimensional undulation, the critical thickness of the solid film, and the critical thickness of the liquid layer are studied, and the stability regions of the solid film have been determined. Emphasis is placed on calculation of critical values, which are the basis of analyzing the stability of the very thin solid film. () 2004 American Institute of Physics. [DOI: 10.1063/1.1648014]
\end{abstract}

\section{INTRODUCTION}

In micro and nano systems, the ratio between the number of atoms at the surface and that in the bulk is much larger than that in macro systems, which accentuates the role of surface forces as well as other surface effects in general. ${ }^{1-3}$ The best-studied example so far involves DerjaguinLandau-Verwey-Overbeek (DLVO) theory consisting of attractive van der Waals ( $\mathrm{vdW}$ ) forces (now extended to include dispersion, induction, and orientation Lifshitz-vdW interactions) $F_{\mathrm{vdW}}$ and repulsive electric double layer (EDL) forces $F_{\mathrm{EDL}}$ in stabilizing colloids. ${ }^{4-7}$

When the thin liquid film is on the solid surface, the stability of the film is fundamental to many phenomena such as dropwise and filmwise condensation, evaporation, and boiling, as well as self-assembly of clusters and molecules. ${ }^{8}$ The intermolecular forces of the interface between the liquid film and solid surface are highly nonlinear. The interplay between these forces at different length scales leads to film dynamics and stability regimes that are not only rich in physics, but also have major engineering implications. The nonlinear dynamical properties and effects on nucleation phenomena have been studied. ${ }^{9,10}$

In the solid-on-liquid structures, however, a thin solid film covers the liquid layer, where the flexural rigidity of the solid film must be considered. The surface energy in solidon-liquid structure is different from that of the liquid film lying on the solid surface. The air-liquid interface is now replaced by the sum of the solid-air interface and solidliquid interface. Since the change of the surface energy at an air-liquid interface is that the surface energy density times

\footnotetext{
a) Author to whom correspondence should be addressed. Fax: +86-1062561284. Electronic mail: yzhao@1nm.imech.ac.cn
}

the area change and the surface energy density is a constant, the energy increases at an air-liquid interface when some liquid molecules inside the bulk emerge to the interface and increase the area. At a solid-liquid interface, however, since the number of atomic sites is fixed and the area change of the interface is due to the stretching or compressing of the interatomic distance, the energy change results from the elastic strain and the surface stress. ${ }^{11-15}$ The surface energy density at an air-liquid interface is positive, which tends to stabilize the liquid layer, whereas at a solid-liquid interface the surface stress can be either positive or negative, which can either stabilize or destabilize the solid film and the liquid layer. ${ }^{16-18}$ Therefore, even a monolayer solid film on a liquid layer can qualitatively change the stability behavior of the liquid layer. Recently, a dimensionless number is introduced to study the stability of a solid film, ${ }^{19}$ which discusses the situation under the action of DLVO.

The classical DLVO theory is a continuum theory that does not consider the discrete molecular nature of the surfaces, solvent, or ions and other factors that can become important at small distances, so it is not surprising that the model breaks down when the liquid medium between two surfaces is only few molecular diameters in width. It has long been postulated that a modified water structure exists at solid-water interfaces. In fact, hydrophobicity is a manifestation of water structure at surfaces. ${ }^{20,21}$ For colloids and interfaces in aqueous media, the predominant effect is attributed to the hydration of the adsorbed counterions and ionic functional groups in the surface. As such surfaces approach each other closely during the interaction, some dehydration of the ions and surface would have to occur, leading to an increase in the free energy and hence a repulsion. These effects are usually referred to as hydration or structural forces. 


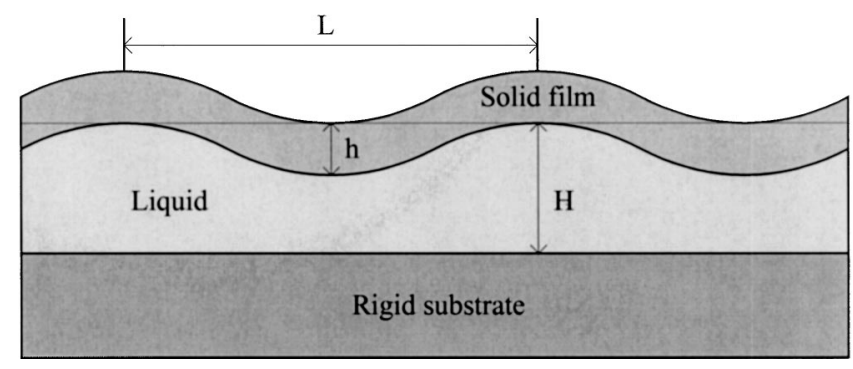

FIG. 1. Schematic diagram of the solid-on-liquid structure.

This explanation, however, is far from being completely understood and theoretically modeled. ${ }^{22}$

Now, in aqueous media, in addition to electrostatic (ionic) and $\mathrm{vdW}$ interactions, there are also the interaction energies contributed by the water molecules. In the case of extremely hydrophilic polar entities, the polar repulsive energies can give rise to a net interfacial repulsion between hydrophilic particles suspended in water. Such net repulsive energies have also been designated as hydration pressure. ${ }^{23}$ They are a consequence of Lewis acid-base interactions between strongly electron-donating particles, immersed in water. ${ }^{24}$ Recently, ${ }^{24}$ the so-called XDLVO has been forwarded, which in addition to classical DLVO interactions, accounts for short-range Lewis acid-base or hydration interactions: significant progress has been made in extending the traditional DLVO model to accommodate these non-DLVO forces-for instance, the structural and steric forces (see the recent review papers in Refs. 25-27).

As illustrated in Fig. 1, this paper studies the solid-onliquid (SOL) structure, which can be fabricated by wafer bonding. Strained SiGe film on a layer of borophosphorosilicate glass is a good application of this structure in optoelectronic aspect. ${ }^{28,29}$ One small perturbation leads to solid film wrinkles, dragging the liquid underneath to flow under the condition that the solid film and liquid layer keep in contact. As aforementioned, Huang and Suo ${ }^{19}$ studied this problem under the action of DLVO forces. Our interest in this problem is focused on the stability analysis of the solid film. Emphasis is placed on the critical wavelength and critical thickness of the solid film and the critical thickness of the liquid layer, which is the basis of analyzing the stability of the solid film. Section II explicitly formulates the total change of free energy in the SOL structure consisting of three parts: respectively, the elastic energy change within the solid film, surface energy, and interaction energy associated with interfacial forces. Besides the DLVO forces, the hydration forces and elastic strain energy due to hydrationinduced layering of liquid molecules close to the solid film surface are also considered because of the hydration forces becoming very strong at short range. Section II also calculates the critical thickness of the solid film, analyzes the critical value of the liquid layer thickness, and discusses how the critical thickness influences the stability of the solid film. The critical wavelength of the solid film under the XDLVO interactions is determined in Sec. III. The stability regimes for the solid film are presented in Sec. IV.

\section{CRITICAL THICKNESS}

\section{A. Expression of free energy change}

The total change of free energy in the solid-on-liquid structure can be written as

$$
\Delta W=\Delta W_{F}+\Delta W_{S}+\Delta W_{V},
$$

where $\Delta W_{F}$ is the elastic energy change within the solid film, $\Delta W_{S}$ is surface component, and $\Delta W_{V}$ is volumetric component.

Assume that the solid film is isotropic and elastic with Young's modulus $E$ and Poisson's ratio $\mu$. Let $x$ and $y$ be the coordinates in the middle plane of the solid film and $z$ perpendicular to the middle plane. Due to the small disturbance, the solid film has a deflection $h(x, y)$, in the $z$ direction. Using the von Karman plate theory, ${ }^{30}$ from the flat state to the wrinkled state, the strain in the film changes by

$$
\Delta \varepsilon=\frac{1}{2}\left(\frac{\partial u}{\partial y}+\frac{\partial v}{\partial x}+\frac{\partial h}{\partial x} \frac{\partial h}{\partial y}\right)-\frac{\partial^{2} w}{\partial x \partial y} z
$$

where $z=0$ at the middle plane and $z= \pm h / 2$ at the top and bottom surfaces of the solid film. The elastic energy within the solid film consists of the in-plane strain energy and the bending energy. From the flat state to the wrinkled state, the change of the elastic energy within the solid film per unit $\operatorname{area}^{19}$ is

$$
\begin{aligned}
\Delta w_{F}= & \frac{\sigma h}{2}\left(\frac{\partial u}{\partial y}+\frac{\partial v}{\partial x}+\frac{\partial h}{\partial x} \frac{\partial h}{\partial y}\right)+\frac{D}{2}\left\{\left(\frac{\partial^{2} h}{\partial x^{2}}+\frac{\partial^{2} h}{\partial y^{2}}\right)^{2}\right. \\
& \left.+2(1-\mu)\left[\left(\frac{\partial^{2} h}{\partial x \partial y}\right)^{2}-\frac{\partial^{2} h}{\partial x^{2}} \frac{\partial^{2} h}{\partial y^{2}}\right]\right\},
\end{aligned}
$$

where $\sigma$ is the in-plane residual stress within the solid film in the state of having no perturbation, $D$ is the flexural rigidity, and $D=E h^{3} / 12\left(1-\mu^{2}\right)$. The parameters $u$ and $v$ are inplane displacements along the $x$ and $y$ directions, respectively.

For simplicity and without loss of generality, just consider the situation of a one-dimensional disturbance, which changes shape to

$$
h^{\prime}(x)=A^{\prime} \cos \frac{2 \pi x}{L}
$$

where $A^{\prime}$ is a small perturbation and $L$ is the wavelength. The elastic energy change of the solid film in one period of the undulation is

$$
\Delta W_{F}=\frac{D A^{\prime 2}}{2}\left(\frac{2 \pi}{L}\right)^{4} \int_{0}^{L} \cos ^{2} \frac{2 \pi x}{L} d x .
$$

By integration, the elastic energy of the solid film in one period of the undulation can be written as

$$
\Delta W_{F}=\frac{4 D A^{\prime 2} \pi^{4}}{L^{3}} \text {. }
$$

To calculate the change of the surface energy, it is necessary to calculate the change of the surface area. Using the cosine profile, the change $\Delta L$ is given as 


$$
\Delta L=\int_{0}^{L} \sqrt{1+\left(\frac{2 \pi A^{\prime}}{L}\right)^{2} \sin ^{2} \frac{2 \pi x}{L}} d x-L .
$$

Due to the small perturbation $A^{\prime} \ll L$, the change $\Delta L$ can be simplified to

$$
\Delta L=2\left(\frac{\pi A^{\prime}}{L}\right)^{2} \int_{0}^{L} \sin ^{2} \frac{2 \pi x}{L} d x=\frac{\left(A^{\prime} \pi\right)^{2}}{L} .
$$

So the change in surface energy in one period of the perturbation $^{10}$ is

$$
\Delta W_{S}=\frac{F\left(A^{\prime} \pi\right)^{2}}{L},
$$

where $F$ is the resultant membrane force of the solid film, $F=\sigma h+f, \sigma$ is the aforementioned residual stress in the solid film in the state of no disturbance, and $f$ is the sum of the surface stresses at the top and bottom surfaces of the solid film.

The energy density is expanded with respect to the disturbance by Taylor series expansion as the following:

$$
\begin{aligned}
w_{L}\left(H+h^{\prime}\right)= & w_{L}(H)+\frac{d w_{L}(H)}{d H} h^{\prime}+\frac{1}{2} \frac{d^{2} w_{L}(H)}{d H^{2}} h^{\prime 2} \\
& +\cdots .
\end{aligned}
$$

The change in volumetric free energy in one period of the undulation can be found as

$$
\begin{aligned}
\Delta W_{L} & =\int_{0}^{L}\left[w_{L}\left(H+h^{\prime}\right)-w_{L}(H)\right] d x \\
& =\int_{0}^{L} \frac{d w_{L}(H)}{d H} h^{\prime} d x+\int_{0}^{L} \frac{1}{2} \frac{d^{2} w_{L}(H)}{d h^{2}} h^{\prime 2} d x+\cdots .
\end{aligned}
$$

Due to mass conservation, the first term on the right side is zero. Using the cosine profile of the film thickness and neglecting terms higher than the second-order term, the volumetric free energy change can be written as

$$
\Delta W_{V}=\frac{A^{\prime 2} L}{4} \frac{d^{2} w_{L}(H)}{d H^{2}} .
$$

\section{B. Volumetric interaction energy}

\section{1. van der Waals forces}

The change of the number of photons in the electromagnetic oscillation modes in a structure made of several media leads to a configurational force, known as the dispersion force or the vdW forces. If the volumetric force interaction between a liquid layer and a solid film in the presence of its vapor is only through vdW interactions, the volumetric interaction energy density can be written as

$$
w_{\mathrm{vdW}}(H)=-\frac{A_{h}}{12 \pi H^{2}},
$$

where $A_{h}$ is Hamaker constant, which depends on the dielectric spectra of the media, and $H$ is the separation gap. When two dissimilar media interact across a film of a third medium, the dispersion force can be either attractive $(A>0)$ or repulsive $(A<0)$. The dispersion force is, however, always attractive when two identical media interact across a film of another medium. The influence of vdW forces on the morphological stability of thin solid films was studied by Refs. 31 and 32

The curvature of the $w_{\mathrm{vdW}}$ has the form

$$
\frac{d^{2} w_{\mathrm{vdW}}(H)}{d H^{2}}=-\frac{A_{h}}{2 \pi H^{4}} \text {. }
$$

\section{Electric double layer}

For charged surfaces in liquid, new phenomena happen mainly as the result of the charge redistribution in the liquid. Basically, the final surface charge is balanced by counterions in the liquid by equal but opposite total charge. The surface electrical potential attracts counterions to the wall and forms a thin layer of immobile ions. Outside the layer, the distribution of the counterions in the liquid mainly follows the exponential decaying dependence away from the surface, which is called the diffuse EDL. EDL has a characteristic length, the Debye length, which depends inversely on the square root of ion concentration in the liquid. ${ }^{8}$ An approximate expression for the interaction energy density can be given as

$$
w_{\mathrm{EDL}}=64 \kappa^{-1} n k_{B} T \beta^{2} \exp (-\kappa H),
$$

where $n$ is the ionic number density, $k_{B}$ is the Boltzmann constant, $T$ is absolute temperature, $\beta$ is given as

$$
\beta=\tanh \left(\frac{z e \phi_{s}}{4 k_{B} T}\right),
$$

where $z$ is the valence of species ions, $\phi_{s}$ is the surface potential, and $e$ is the charge of electrons. Note that the influence of electrostatic forces depends on the Debye length $\kappa^{-1}$, which is given as

$$
\kappa^{-1}=\left(\frac{\varepsilon k_{B} T}{2 n z^{2} e^{2}}\right)^{1 / 2},
$$

where $\varepsilon$ is the dielectric permittivity of the solvent. For a $\mathrm{NaCl}$ solution at room temperature, the Debye length is 30.4 $\mathrm{nm}$ at $10^{-4} \mathrm{~mol}, 0.96 \mathrm{~nm}$ at $0.1 \mathrm{~mol}$, and $0.3 \mathrm{~nm}$ at $1 \mathrm{~mol}$ : in totally pure water at $p \mathrm{H} \mathrm{7,} \mathrm{the} \mathrm{Debye} \mathrm{length} \mathrm{is} 960 \mathrm{~nm}$ or about $1 \mu \mathrm{m}$. Therefore, the EDL forces can have much longer range than vdW forces.

The curvature of the $w_{\mathrm{EDL}}$ can be written as

$$
\frac{d^{2} w_{\mathrm{EDL}}(H)}{d H^{2}}=64 \kappa n k_{B} T \beta^{2} \exp (-\kappa H) \text {. }
$$

Using representative values $\varepsilon=7.21 \times 10^{-10} \mathrm{C}^{2} / \mathrm{Nm}^{2}$ and $T$ $=287 \mathrm{~K}$, we have $\kappa^{-1}=3.041 \mathrm{~nm}$ for a $1: 1$ electrolyte $z$ $=1$ of concentration $0.01 \mathrm{~mol}\left(n=6.02 \times 10^{24} \mathrm{~m}^{-3}\right)$. At a lower electrolyte concentration of $n=6.02 \times 10^{23} \mathrm{~m}^{-3}$, we have $\kappa^{-1}=9.617 \mathrm{~nm}$. The higher electrolyte concentration, the thinner the EDL thickness is.

\section{Hydration force}

There are many aqueous systems where DLVO theory fails and where additional short-range $(<5 \mathrm{~nm})$ exponen- 
tially repulsive solvation forces are observed and commonly referred to as hydration, structural, hydrophilic forces, or non-DLVO forces. Hydration forces arise whenever water molecules bind to surfaces containing hydrophilic groups (i.e., certain ionic, zwitterionic, or H-bonding groups), and then the strength depends on the energy needed to disrupt the ordered water structure and ultimately dehydrate two surfaces as they approach each other.

It is probable that the short-range hydration force between smooth rigid surfaces is always oscillatory, due to the ordered layering of water molecules bound to hydrated surface groups. This may be superimposed on a monotonically repulsive profile due to image interactions and/or a decaying cooperative H-bonding interaction away from the surfaces. It is also likely that for rough or fluid surfaces, the oscillations are smeared out, resulting in a purely monotonic repulsion. It is clear, however, that the strong repulsion between the surfaces and the exponential decay of hydration forces are still detected. The hydration energy density can be written as ${ }^{8}$

$$
w_{\text {Hyd }}=w_{0} \exp \left(-\frac{H}{\lambda}\right) \text {, }
$$

where the decay length $\lambda$ is the order of $1 \mathrm{~nm}$ and $w_{0}$ ranges from 3 to $30 \mathrm{~mJ} / \mathrm{m}^{2}$. Such a relation cannot derived from the first principles but a close fit to experimental observations. It does not account for the oscillatory nature but provides the average energy. However, it should be noted that although Eq. (18a) suggests that hydration energy is asymptotic to a finite value as $H \rightarrow 0$, experimental data show that the hydration energy keeps increasing as $H$ decreases. In view of the fact, it is proposed ${ }^{10}$ that the hydration energy follows

$$
w_{\text {Hyd }}=w_{0}\left(1+\frac{\lambda}{H}\right) \exp \left(-\frac{H}{\lambda}\right) \text {. }
$$

It can be demonstrated that hydration forces dominate over vdW forces and the EDL interaction for $H$ by less than about 3-5 nm.

The curvature of the $w_{H}$ is as follows:

$$
\frac{d^{2} w_{\mathrm{Hyd}}}{d H^{2}}=\frac{w_{0}}{\lambda^{2}}\left(1+\frac{\lambda}{H}+\frac{2 \lambda^{2}}{H^{2}}+\frac{2 \lambda^{3}}{H^{3}}\right) \exp \left(-\frac{H}{\lambda}\right) \text {. }
$$

\section{Elastic strain energy}

If the lattice constant of the solid film is not equal to that of the layered icelike water, the bonds between the water molecules in the hydration-induced layered structure close to the solid surface will be strained. The strain has an influence on the phenomena at the solid-liquid interface. Recent experiments have also shown that strain energy can play a significant role in nucleation processes, especially for the heterogeneous nucleation of ice from water on a solid surface. ${ }^{33-35}$ In addition, a recent experiment showed that the first few layers of liquid mercury in contact with a diamond surface form a layered structure with the orientation of solid $\mathrm{Hg}$ crystals. ${ }^{36}$ If the first layers are solid like, there should be some strain due to the mismatch in the lattice constants of the solidlike liquid layer in contact with the solid film. It is proposed that the strain energy density in the liquid layer is as follows:

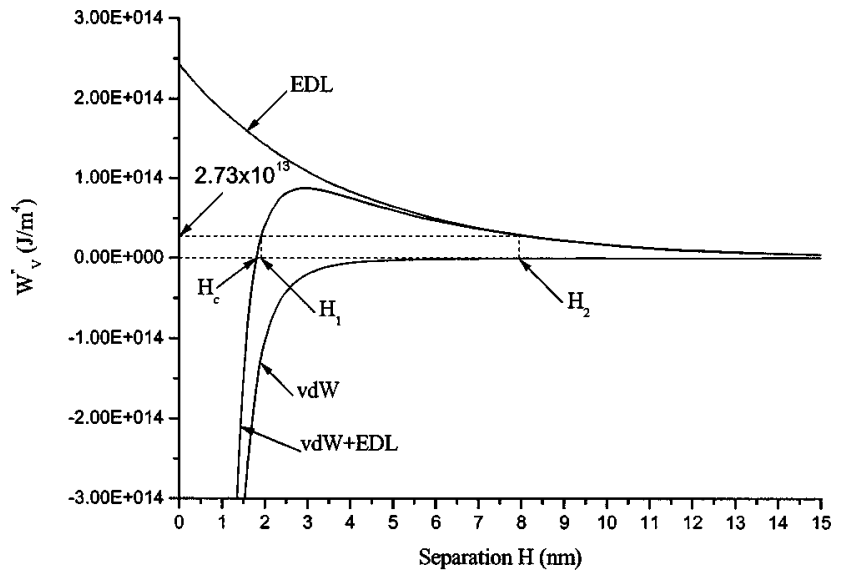

FIG. 2. Curvature of the DLVO interaction energy as a function of separation $H$.

$$
w_{\mathrm{ESE}}=\frac{E \lambda \varepsilon_{0}^{2}}{2}\left[1-\exp \left(-\frac{H}{\lambda}\right)\right],
$$

where $\lambda$ is the decay length and $\varepsilon_{0}$ is the strain of the first monolayer, which typically ranges between 0 and 0.04 and arises from lattice mismatch.

The curvature of the $w_{\mathrm{ESE}}$ is given as

$$
\frac{d^{2} w_{\mathrm{ESE}}(H)}{d H^{2}}=-\frac{E \varepsilon_{0}^{2}}{2 \lambda} \exp \left(-\frac{H}{\lambda}\right) \text {. }
$$

\section{Critical thickness of the liquid layer and solid film}

\section{Critical thickness of the liquid layer by XDLVO theory}

From the calculation in Sec. I, if only the DLVO forces (attractive vdW force and repulsive EDL) are considered, the sum of curvature of the volumetric free energy can be written as

$$
\left(\frac{d^{2} w_{V}}{d H^{2}}\right)_{\text {DLVO }}=-\frac{A_{h}}{2 \pi H^{4}}+64 \kappa n k_{B} T \beta^{2} \exp (-\kappa H) .
$$

From Eq. (12), the curvature of vdW forces is proportional to the Hamaker constant $A_{h}$, inversely proportional to $H^{4}$. Therefore, the curvature of the vdW forces is more sensitive to $H$ than to $A_{h}$. The curvature of the electric double layer is exponentially decaying with $H$.

Figure 2 plots the curvature of vdW, EDL, and the combination of the two interactions. When the thickness of the liquid layer is thick, the effect of the interaction energy for the curvature is very small, even can be neglected, whereas when the liquid layer thickness is less than $10 \mathrm{~nm}$, the effect of the interaction energy cannot be neglected any more and becomes significant. Figure 2 shows the critical thickness $H_{c}$ resulting from the DLVO interactions and the critical thickness of the liquid layer is approximately $2 \mathrm{~nm}$.

At small separation, below a few molecular diameters, some properties such as the dielectric constant and density are no longer the same as in the bulk, and a short-distance intermolecular pair potential can be quite different from continuum theories, where DLVO theories are regarded as con- 
tinuum theories. These short-distance interactions are usually referred to as solvation forces, whereas when the medium is water, these forces are named hydration forces. Hydration forces depend not only on the property of the intervening medium but also on the chemical and physical properties of the surfaces, whether amorphous or crystalline, smooth or rough, rigid or fluid like. Such forces can be very strong at short range, and they are particularly important for determining the magnitude of the adhesion between the two surfaces or interaction energy.

When the attractive vdW, repulsive EDL, and hydration forces $[\mathrm{XDLVO}(3)]$ are considered, the curvature sum can be given as

$$
\begin{aligned}
\left(\frac{d^{2} w_{V}}{d H^{2}}\right)_{\mathrm{XDLVO}(3)}= & -\frac{A_{h}}{2 \pi H^{4}}+64 \kappa n k_{B} T \beta^{2} \exp (-\kappa H) \\
& +\frac{w_{0}}{\lambda^{2}}\left(1+\frac{\lambda}{H}+\frac{2 \lambda^{2}}{H^{2}}+\frac{2 \lambda^{3}}{H^{3}}\right) \\
& \times \exp \left(-\frac{H}{\lambda}\right) .
\end{aligned}
$$

Figure 3 depicts the curve of $d^{2} w_{V} / d H^{2}$ versus separation $H$. Since hydration forces play an important role at the small separation, it must be considered in solid-on-liquid structures. As shown in Fig. 3, the hydration force is very large at separation below $3 \mathrm{~nm}$. The sum of the curvature of the XDLVO(3) forces is plotted. From the Figs. 2 and 3(b), we can see that the curve $\left(d^{2} w_{V} / d H^{2}\right)_{\mathrm{XDLVO}(3)}$ moves to the left in contrast to that of $\left(d^{2} w_{V} / d H^{2}\right)_{\text {DLVO }}$ and the critical thickness of the former is smaller than that of the latter.

It is important to note that hydration forces do not arise simply because liquid molecules tend to structure into semiordered layers at surfaces. They arise because of the disruption or change of this ordering during the approach of a second surface. If there were no change, there would be no hydration forces, whereas the two effects are of course related, the greater the tendency towards structuring at an isolated surface, the greater the hydration forces between the two surfaces. In solid-on-liquid structures, the bonds between the water molecules in the hydration-induced layered structure close to the solid film surface will be strained if the lattice constant of the solid film is not equal to that of the layered icelike water. In fact, the strain affects the phenomena at the solid-liquid interface. So there should be some strain because of the mismatch in the lattice constant of the solidlike liquid layer in contact with the solid film if the first few layers are solidlike. Note that there is no strain between the water molecules in the bulk water. Recent experiments have also shown that strain energy can play a significant role in the nucleation of ice from water on a solid surface.

When all these four terms-i.e., vdW, EDL, hydration, and elastic strain energy $[\mathrm{XDLVO}(4)]$ - are considered, the curvature sum of the volumetric free energy can be written as
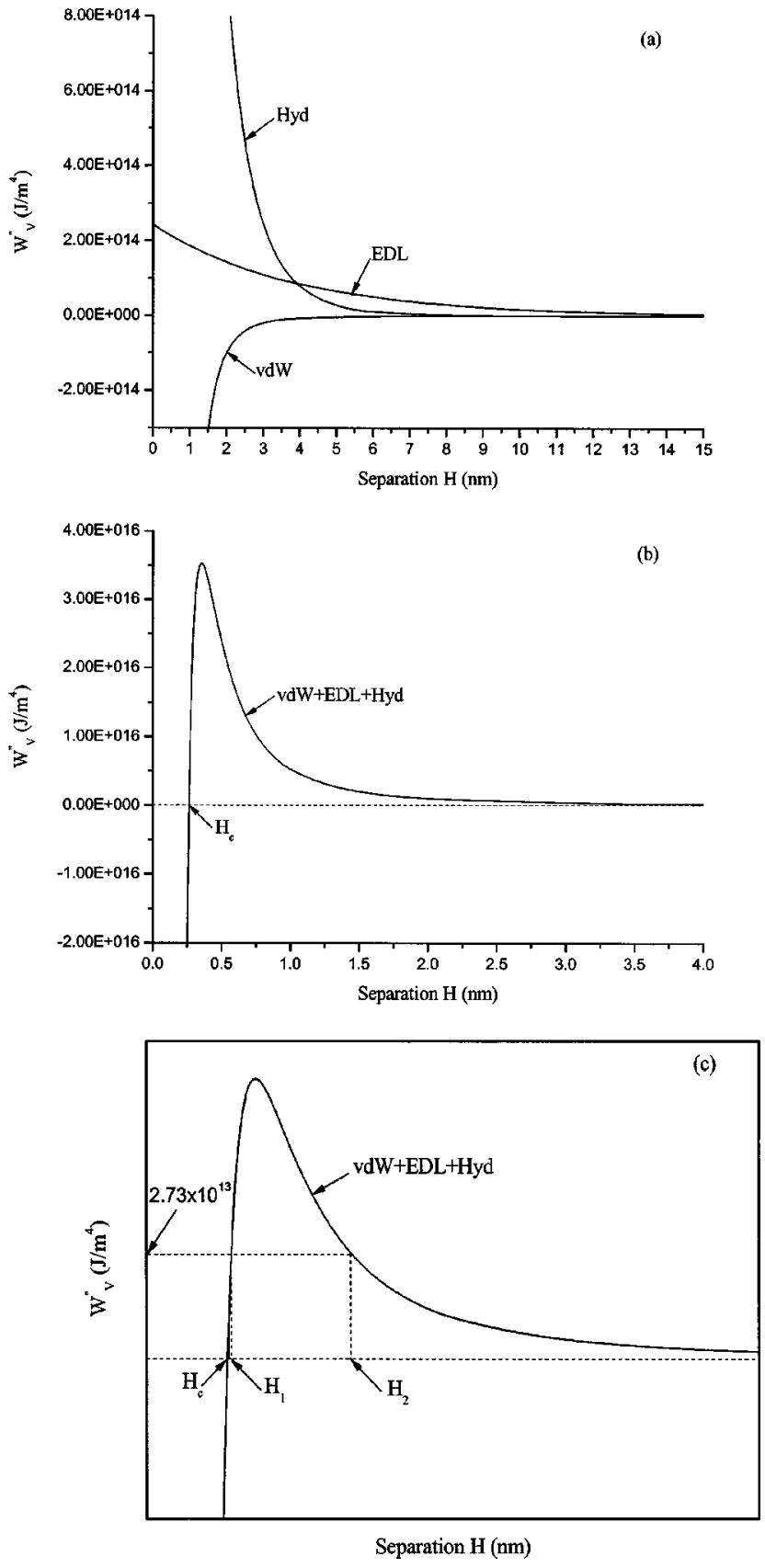

FIG. 3. (a) Curvature of the XDLVO interaction energies as a function of separation $H$. (b) Resultant curvature of the XDLVO interaction energies as a function of separation $H$. (c) Schematic curvature profile of the XDLVO interaction energies.

$$
\begin{aligned}
\left(\frac{d^{2} w_{V}}{d H^{2}}\right)_{\mathrm{XDLVO}(4)}= & {\left[\frac{w_{0}}{\lambda^{2}}\left(1+\frac{\lambda}{H}+\frac{2 \lambda^{2}}{H^{2}}+\frac{2 \lambda^{3}}{H^{3}}\right)-\frac{E \varepsilon_{0}^{2}}{2 \lambda}\right] } \\
& \times \exp \left(-\frac{H}{\lambda}\right)-\frac{A_{h}}{2 \pi H^{4}} \\
& +64 \kappa n k_{B} T \beta^{2} \exp (-\kappa H) .
\end{aligned}
$$

Figure 4(b) shows the critical thickness $H_{c}$ resulting from the $\mathrm{XDLVO}(4)$ interactions, respectively. It is clear from Figs. 2(b) and 4(b) that the critical thickness under the DLVO interactions is not same as that in the state of XDLVO interactions. Hence the effect of the XDLVO, especially the hy- 

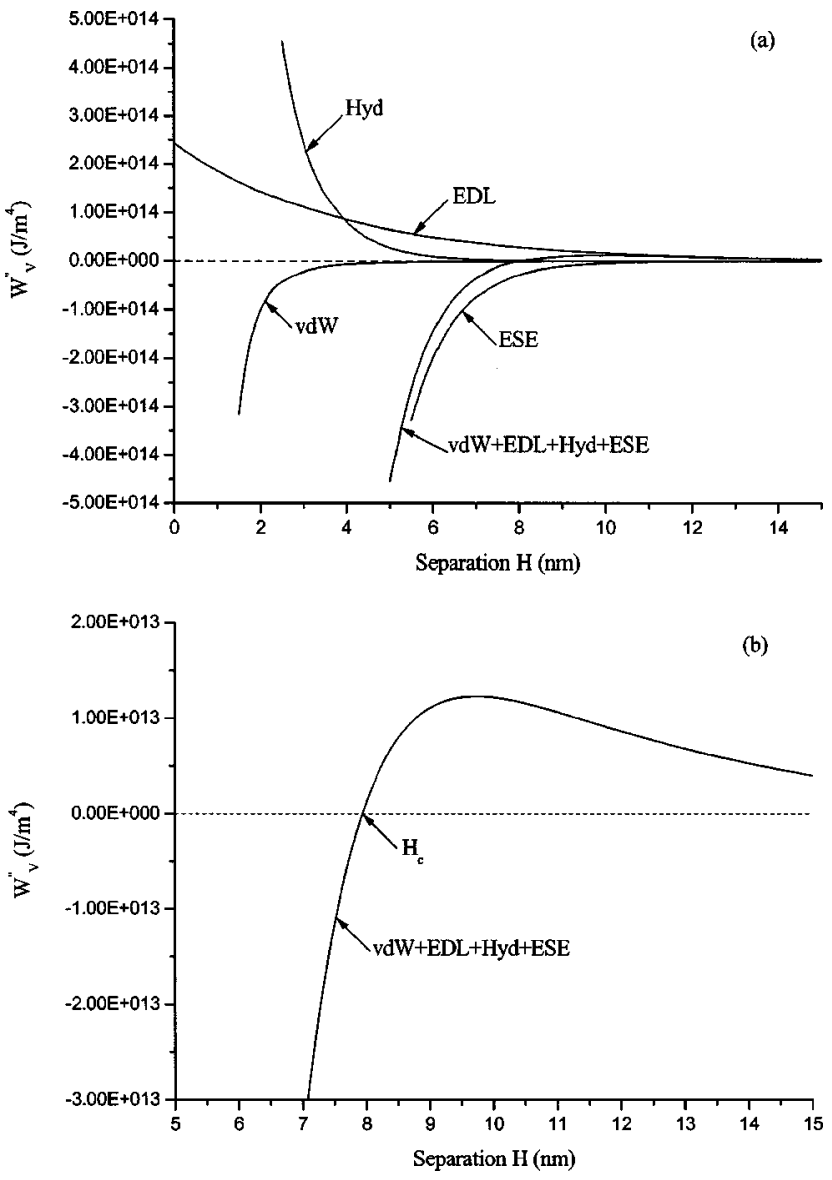

FIG. 4. (a) Curvature of the interaction energy as a function of separation $H$ in the presence of $\mathrm{vdW}$, EDL, hydration force, and elastic strain energy. (b) Resultant curvature of the interaction energy as a function of separation $H$ in the presence of these four interactions.

dration force and resulting elastic strain energy, cannot be neglected. The value of the critical thickness of the liquid layer is independent of the solid film thickness $h$. As shown in Fig. 4(b), the critical thickness is about $8 \mathrm{~nm}$. However, the critical thickness by DLVO theory is about $1.8 \mathrm{~nm}$, as shown in Fig. 2.

\section{Critical thickness of the solid film}

The total change of free energy in the solid-on-liquid structure is given as

$$
\begin{aligned}
\Delta W_{V}= & \frac{A^{\prime 2} L}{4}\left\{\left[-\frac{A_{h}}{2 \pi H^{4}}+64 \kappa n k_{B} T \beta^{2} \exp (-\kappa H)\right.\right. \\
& -\frac{E \varepsilon_{0}^{2}}{2 \lambda} \exp \left(-\frac{H}{\lambda}\right)+\frac{w_{0}}{\lambda^{2}}\left(1+\frac{\lambda}{H}+\frac{2 \lambda^{2}}{H^{2}}+\frac{2 \lambda^{3}}{H^{3}}\right) \\
& \left.\left.\times \exp \left(-\frac{H}{\lambda}\right)\right]+\frac{16 D \pi^{4}}{L^{4}}+\frac{4 F \pi^{2}}{L^{2}}\right\} .
\end{aligned}
$$

Let $a=4 E \pi^{4} / 3 L^{4}\left(1-\mu^{2}\right), \quad b=4 \sigma \pi^{2} / L^{2}$, and $f=0$ : the stability analysis of the solid film is as follows.

(1) When the membrane force is tensile, $16 D \pi^{4} / L^{4}$ $+4 F \pi^{2} / L^{2}=a h^{3}+b h>0$, the solid film is always stable if the liquid thickness is greater than the critical thickness $H_{c}$, whereas the solid film is unstable if the liquid thickness is less than the critical thickness $H_{c}$ and $a h^{3}+b h$ $<\left|\left(d^{2} w_{V} / d H^{2}\right)_{\mathrm{XDLVO}(4)}\right|$ and stable if the liquid thickness is less than the critical thickness $H_{c}$ and $a h^{3}+b h$ $>\left|\left(d^{2} w_{V} / d H^{2}\right)_{\mathrm{XDLVO}(4)}\right|$.

(2) When the membrane force is compressive, there exists a critical thickness of the solid film $h_{c}$. Let $16 D \pi^{4} / L^{4}$ $+4 F \pi^{2} / L^{2}=a h^{3}+b h=0$ : the critical thickness of the solid film can be calculated as $h_{c}=\sqrt{-3 L^{2}\left(1-\mu^{2}\right) \sigma / E \pi^{2}}$. When $h>h_{c}$, the solid film is stable if $H>H_{c}$, whereas the solid film is stable if $H<H_{c}$ and $a h^{3}+b h$ $>\left|\left(d^{2} w_{V} / d H^{2}\right)_{\operatorname{XDLV}(4)}\right|$ and unstable if $H<H_{c}$ and $a h^{3}$ $+b h<\left|\left(d^{2} w_{V} / d H^{2}\right)_{\mathrm{XDLVO}(4)}\right|$. When $h<h_{c}$, the solid film is unstable if $H<H_{c}$, whereas the solid film is stable if $H$ $>H_{c}$ and $\left(d^{2} w_{V} / d H^{2}\right)_{\mathrm{XDLVO}(4)}>\left|a h^{3}+b h\right|$ and unstable if $H>H_{c}$ and $\left(d^{2} w_{V} / d H^{2}\right)_{\operatorname{XDLV}(4)}<\left|a h^{3}+b h\right|$.

Since the stability of a solid film depends on the value of $\Delta W_{V}$, when $\Delta W_{V}>0$, the change of total volumetric energy due to small perturbations is greater than zero and the solid film is stable, whereas when $\Delta W_{V}<0$, the solid film is unstable.

\section{CRITICAL WAVELENGTH OF THE SOLID FILM}

The total change of free energy in the solid-on-liquid structure can be written as

$$
\left(\Delta W_{V}\right)_{\mathrm{XDLVO}(4)}=\frac{A^{\prime 2}}{L^{3}}\left(A L^{4}+B L^{2}+C\right),
$$

where $A, B, C$ are

$$
\begin{aligned}
A= & \frac{1}{4}\left\{-\frac{A_{h}}{2 \pi H^{4}}+64 \kappa n k_{B} T \beta^{2} \exp (-\kappa H)\right. \\
& \left.+\left[\frac{w_{0}}{\lambda^{2}}\left(1+\frac{\lambda}{H}+\frac{2 \lambda^{2}}{H^{2}}+\frac{2 \lambda^{3}}{H^{3}}\right)-\frac{E \varepsilon_{0}^{2}}{2 \lambda}\right] \exp \left(-\frac{H}{\lambda}\right)\right\}, \\
B= & F \pi^{2}, \\
C & =4 D \pi^{2} .
\end{aligned}
$$

For the flat film to be unstable, the total change in free energy must be less than zero- that is, $\Delta W_{V}<0$. By letting $\Delta W_{V}<0$, the inequality about the wavelength $L$ can be written as

$$
A L^{4}+B L^{2}+C<0 .
$$

If $B^{2}-4 A C=0$, then

$$
\begin{aligned}
F^{2}- & D\left\{-\frac{A_{h}}{2 \pi H^{4}}+64 \kappa n k_{B} T \beta^{2} \exp (-\kappa H)\right. \\
& \left.+\left[\frac{w_{0}}{\lambda^{2}}\left(1+\frac{\lambda}{H}+\frac{2 \lambda^{2}}{H^{2}}+\frac{2 \lambda^{3}}{H^{3}}\right)-\frac{E \varepsilon_{0}^{2}}{2 \lambda}\right] \exp \left(-\frac{H}{\lambda}\right)\right\}=0 .
\end{aligned}
$$

That is, the curvature of volumetric free energy can be written as

$$
\frac{d^{2} w_{V}}{d H^{2}}=\frac{F^{2}}{D} .
$$

So $F^{2} / D$ is the critical curvature. Under the condition of $B^{2}-4 A C<0$, the total free energy change $\Delta W_{V}=A L^{4}$ 
$+B L^{2}+C<0$ if $A<0$ and $\Delta W_{V}=A L^{4}+B L^{2}+C>0$ if $A$ $>0$ : that is, the solid film is unstable if $A<0$ and stable if $A>0$ in the state of $B^{2}-4 A C<0$.

Since the parameter $C$ is always greater than zero, three cases are distinguished in following discussion, which is on the condition of $C>0$.

Case 1: $B<0$

(1) When $A<0$, critical wavelength can be written as

$$
L_{c r}=\sqrt{\frac{-B-\sqrt{B^{2}-4 A C}}{2 A}} .
$$

The flat film is unstable if $L>L_{c r}$ and stable if $0<L<L_{c r}$.

(2) When $A=0$, critical wavelength can be written as

$$
L_{c r}=\sqrt{-\frac{C}{B}} .
$$

The flat film is unstable if $L>L_{c r}$ and stable if $0<L<L_{c r}$.

(3) When $A>0$, there exist two critical wavelengths, which can be written as

$$
\begin{aligned}
& L_{c r 1}=\sqrt{\frac{-B-\sqrt{B^{2}-4 A C}}{2 A}}, \\
& L_{c r 2}=\sqrt{\frac{-B+\sqrt{B^{2}-4 A C}}{2 A}} .
\end{aligned}
$$

It is obvious that $L_{c r 1}<L_{c r 2}$. So the flat film is unstable if $L_{c r 1}<L<L_{c r 2}$, stable if $0<L<L_{c r 1}$ or $L>L_{c r 2}$.

Case 2: $B>0$

(1) When $A<0$, critical wavelength can be written as

$$
L_{c r}=\sqrt{\frac{-B-\sqrt{B^{2}-4 A C}}{2 A}} .
$$

The flat film is unstable if $L>L_{c r}$ and stable if $0<L<L_{c r}$.

(2) When $A=0$, the total change of free energy in the solid-on-liquid structure is greater than zero-that is, $A L^{4}$ $+B L^{2}+C>0$. The flat film is always stable.

(3) When $A>0$, the total change of free energy in the solid-on-liquid structure is greater than zero-namely, $A L^{4}$ $+B L^{2}+C>0$. The flat film is always stable.

Case 3: $B=0$

(1) When $A<0$, the critical wavelength can be written as

$$
L_{c r}=\left(-\frac{C}{A}\right)^{1 / 4} \text {. }
$$

The flat film is unstable if $L>L_{c r}$ and stable if $0<L<L_{c r}$.

(2) When $A \geqslant 0$, the total change of free energy in the solid-on-liquid structure is greater than zero-that is, $A L^{4}$ $+B L^{2}+C>0$. The flat film is always stable.

\section{DISCUSSION}

We will use the representative values $A_{h}=10^{-20} \mathrm{~J}, \phi_{s}$ $=0.1 \mathrm{~V}, E=100 \mathrm{GPa}, \mu=0.3$, and $\sigma= \pm 100 \mathrm{MPa}$ in this section for the calculations.

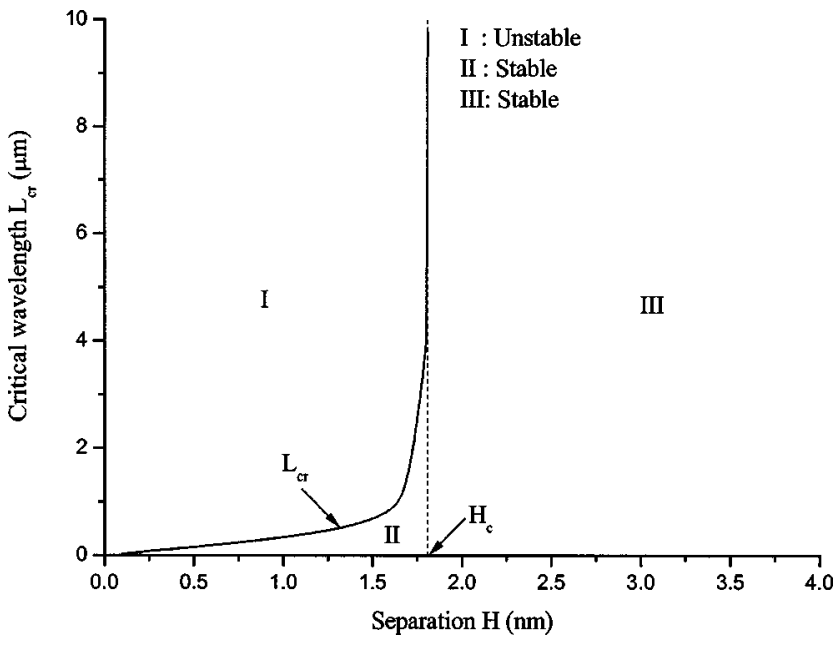

FIG. 5. The critical wavelength $L_{c r}$ profile versus the separation distance $H$ when DLVO theory is applied. The stability regime is plotted when the membrane force is tensile.

\section{A. Critical thickness}

As shown in Fig. 2, vdW forces predominate over the EDL before the resultant curve arrives at the maximum, whereas after the maximum, the EDL is predominant. The curvature of the combined interaction energy is concave down for the thin liquid layer, but concave up for thick liquid layer, and the critical thickness of the liquid layer is about $1.8 \mathrm{~nm}$.

Figure 3 shows the curvature of the $\mathrm{XDLVO}(3)$ interaction energy. VdW forces predominate over EDL and hydration forces, whereas after the maximum, the EDL and hydration forces are predominant. Since hydration forces are particularly important when the separation distance is smaller than $3 \mathrm{~nm}$, the hydration forces predominate over the EDL after the maximum. The curvature of the combined interaction energy is concave down for the thin liquid layer, but concave up for the thick liquid layer, and the critical thickness of the liquid layer is about $0.26 \mathrm{~nm}$, which attributes the action of hydration forces.

$\mathrm{vdW}$ forces and elastic strain energy predominate over EDL and hydration forces before the resultant curve arrives at the maximum, as shown in Fig. 4: however, the EDL and hydration forces are predominant after the maximum. The curvature of the combined interaction energy is concave down for the thin liquid layer, but concave up for the thick liquid layer, and the critical thickness of the liquid layer is about $8 \mathrm{~nm}$.

\section{B. Stability of the solid film}

When the membrane force is tensile, using representative values as before and $E=100 \mathrm{GPa}, \mu=0.3, \quad \sigma$ $=100 \mathrm{MPa}, h=10 \mathrm{~nm}$, and $f=0$, the stability regimes are plotted in Figs. 5-7 under the condition of different combinations of interaction energies.

When the interaction energy result from DLVO theory, the stability regime is plotted in Fig. 5 under the condition that the membrane force be tensile. As shown in Fig. 5, in region I, $L>L_{c r}$, and the flat film is unstable. In region II, 


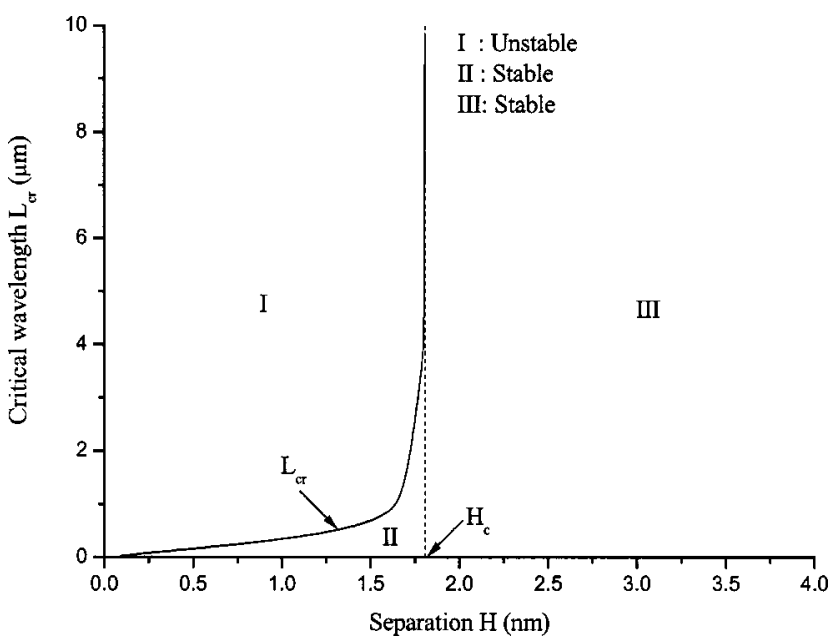

FIG. 6. The critical wavelength $L_{c r}$ profile versus the separation distance $H$ when DLVO theory and hydration force are applied. The stability regime is plotted when the membrane force is tensile.

$0<L<L_{c r}$, and the flat film is stable. In region III, $\Delta W_{V}$ $=A L^{4}+B L^{2}+C>0$, and the flat film is always stable. That is, when the membrane force is tensile, the flat film is stable if $H>H_{c}$. But there exists a critical wavelength if $H<H_{c}$ : the flat film is unstable if $L>L_{c r}$ and stable if 0 $<L<L_{\text {cr }}$.

When XDLVO(3) interaction energies are considered, Fig. 6 shows the stability regime under the condition that the membrane force be tensile. In region I, $L>L_{c r}$, and the flat film is unstable. In region II, $0<L<L_{c r}$, and the flat film is stable. In region III, the change of free energy is greater than zero, $\Delta W_{V}>0$, so the flat film is always stable. That is, the flat film is stable if $H>H_{c}$ when the membrane force is tensile, and the flat film is unstable if $L>L_{c r}$ and $H<H_{c}$ and stable if $0<L<L_{c r}$ and $H<H_{c}$.

When $\mathrm{XDLVO}(4)$ interaction energies are considered, the stability regime is shown in Fig. 7 under the condition that the membrane force be tensile. In region I, $L>L_{c r}$, and

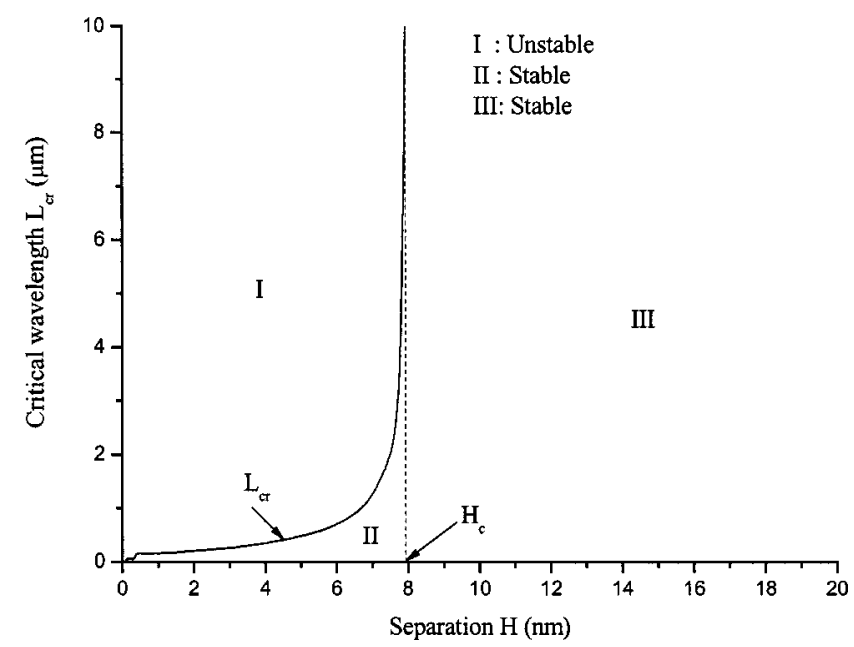

FIG. 7. The critical wavelength $L_{c r}$ profile versus the separation distance $H$. Interaction energies that were considered for this calculation were $\mathrm{vdW}$, EDL, hydration force, and elastic strain energy. The stability regime is plotted in when the membrane force is tensile. the flat film is unstable. In region II, $0<L<L_{c r}$, and the flat film is stable. In region III, the flat film is always stable because of the total free energy change $\Delta W_{V}=A L^{4}+B L^{2}$ $+C>0$. That is, when the membrane force is tensile, the flat film is stable if $H>H_{c}$. But there exists a critical wavelength if $H<H_{c}$ : the flat film is unstable if $L>L_{c r}$ and stable if $0<L<L_{c r}$. From comparison of the three cases, we can see that the magnitude of the critical thickness of the liquid layer, $H_{c}$, is different, but the distribution trend of the stability regime is identical.

When the membrane force is compressive, using the representative values as before and $E=100 \mathrm{GPa}, \mu=0.3, \sigma=$ $-100 \mathrm{MPa}, h=10 \mathrm{~nm}$, and $f=0$, the value of $F^{2} / D$ approximates $2.73 \times 10^{13} \mathrm{~J} / \mathrm{m}^{4}$. This value is denoted in Fig. 2 . We make a line parallel to the axis of separation $H$ from this point of $F^{2} / D$, and this line and the curve of resultant interactions intersect with two points. Then we make perpendicular lines from the two points, respectively, which intersects with the axis of separation $H$ at two points of $H_{1}$ and $H_{2}$. Since the value of $F^{2} / D$ is less than the maximum of the resultant curve of these three interactions, the line parallel to axis of separation $H$ from $F^{2} / D$ intersects with the curve of resultant interactions at two points. So according to the same method as above, the points of $H_{1}$ and $H_{2}$ are obtained from Fig. 3. However, when the XDLVO(4) forces are considered, the value of $F^{2} / D$ exceeds the maximum of the resultant curve of these four interactions, and so there are no intersections with the curve of the resultant interactions.

When the intermolecular forces result from DLVO theory, the stability regime is potted in Fig. 8. Since the critical thickness $H_{c}$ is very near $H_{1}$, two figures are plotted in order to explicitly express the stability regimes between $H_{c}$ and $H_{1}$. In region I, we have $L>L_{c r 1}$, and the flat film is unstable. In region II, $0<L<L_{c r 1}$, the flat film is stable. In region III, we have $L>L_{c r 2}$, and the flat film is stable. In region IV, $0<L<L_{c r 1}$, and the flat film is stable. In region $\mathrm{V}, L_{c r 1}<L<L_{c r 2}$, and the flat film is unstable. In region VI, $H_{1}<H<H_{2}$, and the flat film is stable. In region VII, $L$ $>L_{c r 2}$, and the flat film is stable. In region VIII, $0<L$ $<L_{c r 1}$, and the flat film is stable. In region IX, $L_{c r 1}<L$ $<L_{c r 2}$, and the flat film is unstable.

When XDLVO(3) intermolecular forces are considered, Fig. 9 shows the stability regime. Since the distance between $H_{c}$ and $H_{1}$ in Fig. 9 is shorter than that in Fig. 8, the stability regime between 0 and $20 \mathrm{~nm}$ is divided into three parts. In region I, $L>L_{c r 1}$, and the flat film is unstable. In region II, $0<L<L_{c r 1}$, the flat film is stable. In region III, $L>L_{c r 2}$, and the flat film is stable. In region IV, $0<L<L_{c r 1}$, and the flat film is stable. In region $\mathrm{V}, L_{c r 1}<L<L_{c r 2}$, and the flat film is unstable. In region VI, $H_{1}<H<H_{2}$, and the flat film is stable. In region VII, $L>L_{c r 2}$, and the flat film is stable. In region VIII, $0<L<L_{c r 1}$, and the flat film is stable. In region IX, $L_{c r 1}<L<L_{c r 2}$, and the flat film is unstable. The critical thickness $H_{c}$ of the liquid layer is indicated in this regime, as do $H_{1}$ and $H_{2}$.

For DLVO(4), there do not exist the critical values of $H_{1}$ and $H_{2}$ because of the value of $F^{2} / D$ greater than the maximum of the resultant curve of these four interactions. As shown in Fig. 10, five different regions can be partitioned. 

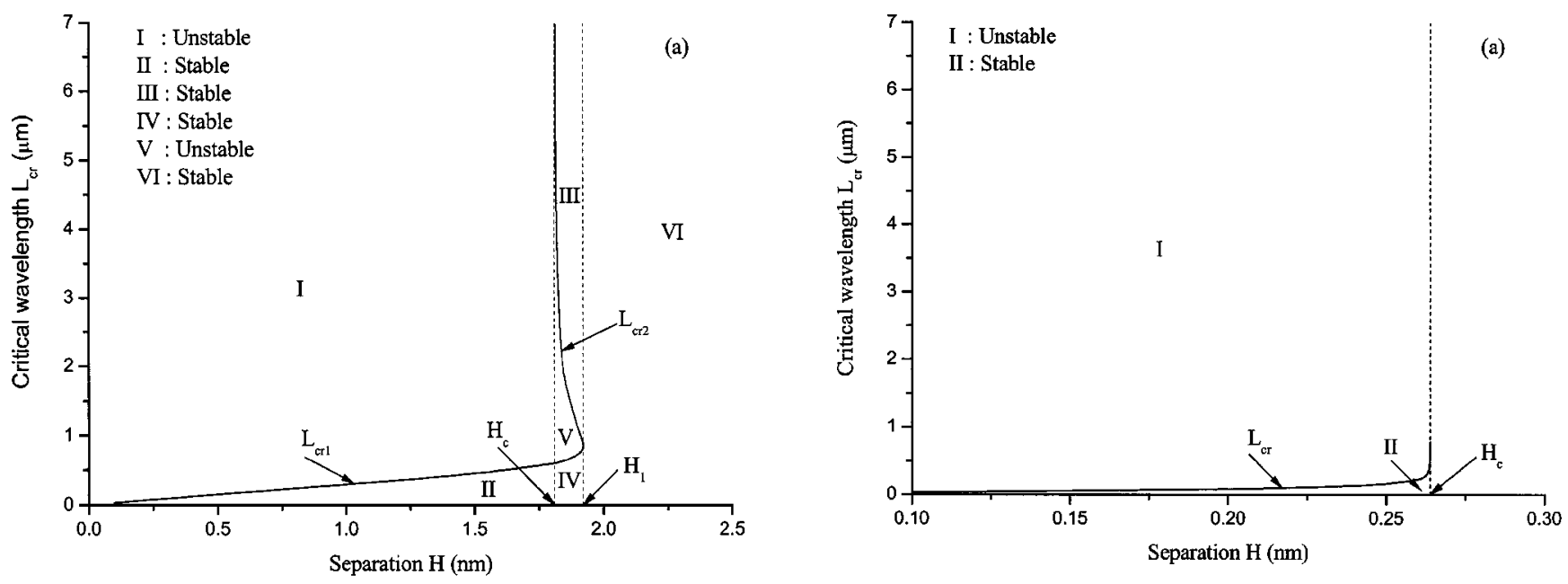

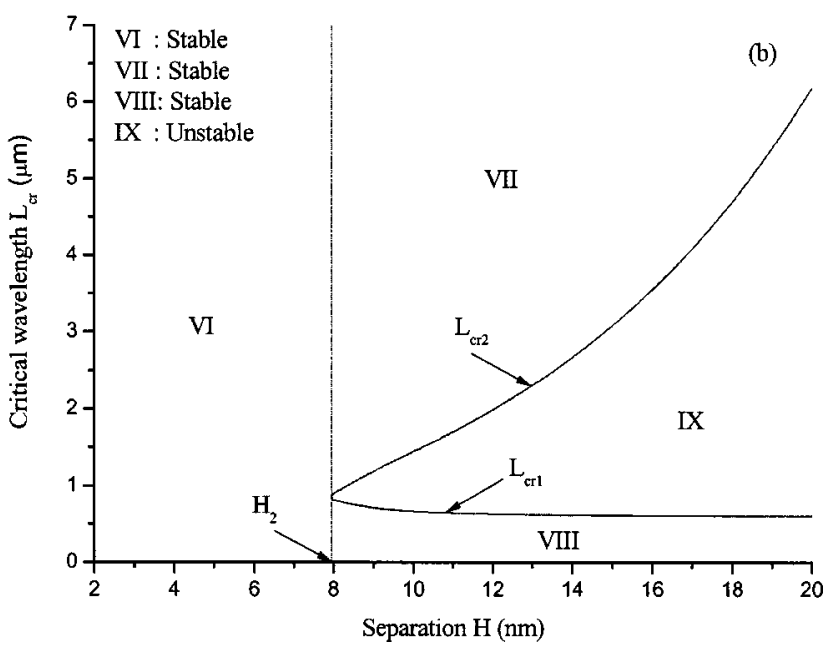

FIG. 8. Stability regime of the solid film in terms of the critical wavelength $L_{c r}$ as a function of separation $H$ by using DLVO theory. (a) Stability regime between 0 and $2.5 \mathrm{~nm}$. (b) Stability regime between $2 \mathrm{~nm}$ and $20 \mathrm{~nm}$.

The flat film is unstable in regions I and V: inequalities $L$ $>L_{c r 1}$ and $L_{c r 1}<L<L_{c r 2}$ are held in these two regions, respectively. The flat film is stable in regions II, III, and IV: inequalities $0<L<L_{c r 1}, L>L_{c r 2}$, and $0<L<L_{c r 1}$ are held in these three regions, respectively. The critical thickness of the liquid layer, $H_{c}$, is indicated by the dotted line.

\section{SUMMARY}

The critical wavelength and critical thickness of the solid film are calculated, and the critical thickness of the liquid layer is obtained in the presence of elastic energy change within the solid film, surface component, and volumetric component consisting of XDLVO(4). A one-dimensional disturbance has been performed to study the stability of very thin solid-on-liquid structures. The solid film is stable when the total change of free energy in the SOL structure is greater than zero, whereas the solid film is unstable when the total change of free energy in the SOL structure is less than zero. Stability regimes of the solid film are plotted under different interactions. It is shown that tensile residual stress of the solid film tends to stabilize the film, vdW forces with positive Hamaker constant, and elastic strain energy destabiliza-
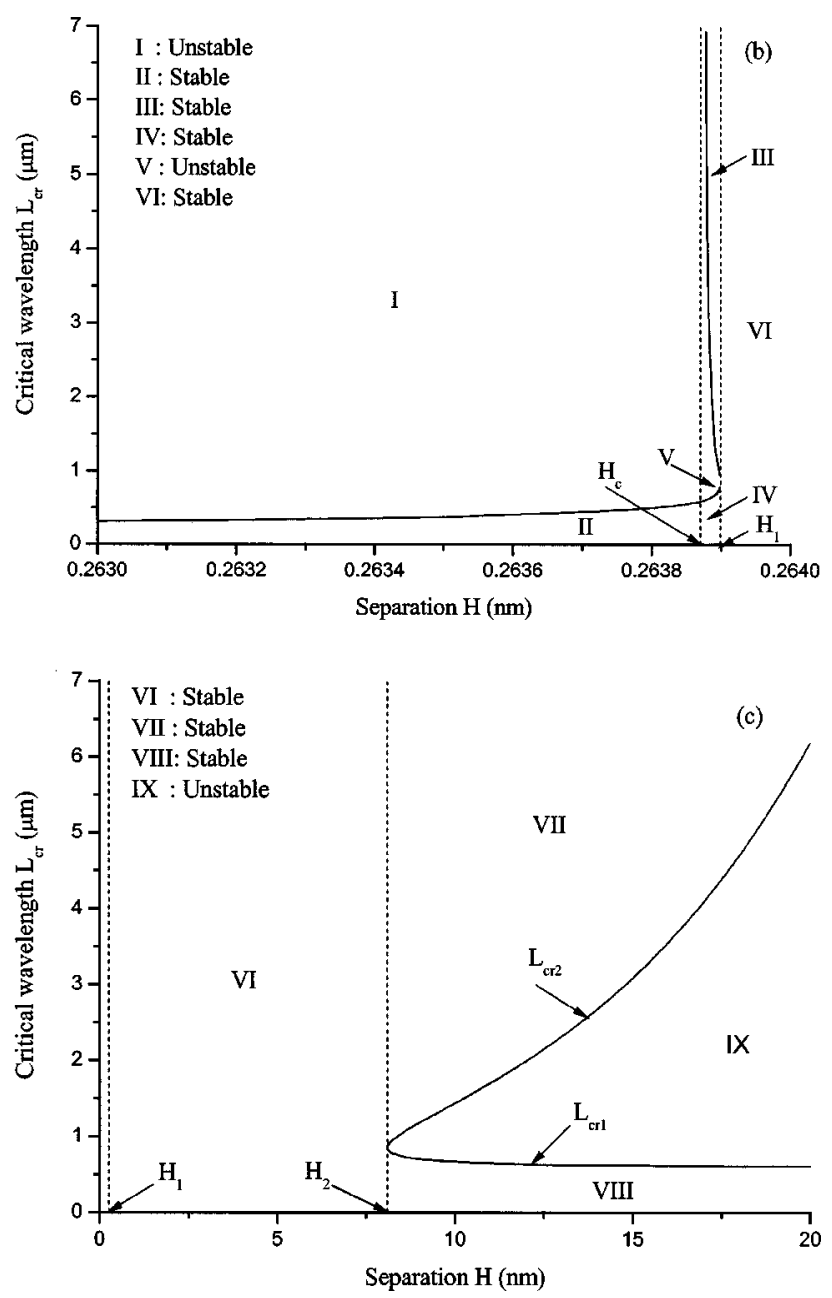

FIG. 9. Stability regime of the solid film in terms of the critical wavelength $L_{c r}$ as a function of separation $H$ by using $\operatorname{XDLVO(3).~(a)~Stability~regime~}$ between 0 and $H_{c}$. (b) Stability regime between $H_{c}$ and $H_{1}$. (c) Stability regime between $H_{c}$ and $20 \mathrm{~nm}$.

tion of the film: however, vdW forces with negative Hamaker constant, EDL, and hydration forces stabilize the film. The effect of critical wavelength and the critical thickness on the stability of the solid film are estimated. As far as XDLVO(4) is concerned, there exists only a critical wavelength $L_{c r 1}$ when the separation lies between 0 and $H_{c}$-the thin solid film is unstable if $L>L_{c r 1}$ and stable if 


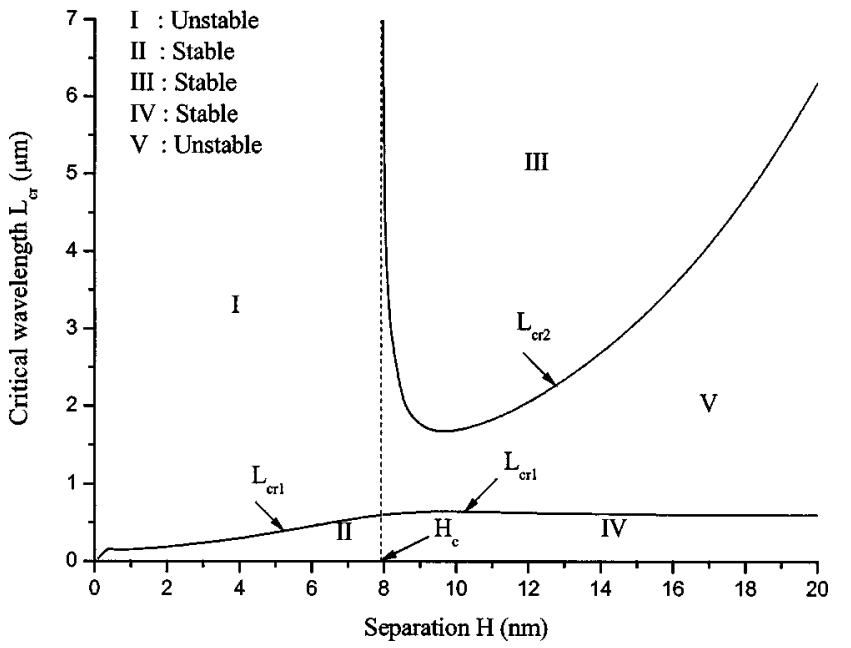

FIG. 10. Stability regime of the solid film in terms of the critical wavelength $L_{c r}$ as a function of separation $H$. Interaction energies for this calculation were vdW, EDL, hydration force, and elastic strain energy.

$0<L<L_{c r 1}$-whereas there exist two critical wavelengths of $L_{c r 1}$ and $L_{c r 2}$ when the separation is greater than $H_{c}$ - the thin solid film is unstable if $L_{c r 1}<L<L_{c r 2}$ and stable if 0 $<L<L_{c r 1}$ and $L>L_{c r 2}$. When the ion concentration of the liquid layer becomes thicker, the critical thickness of the liquid layer becomes smaller.

\section{ACKNOWLEDGMENTS}

The authors acknowledge financial support of the Chinese Academy of Sciences (Grant No. KJCX2-SW-L2) and the National Natural Science Foundation of China (Grant Nos. 10225209, 50131160739, and 10072068).

\section{APPENDIX}

The nomenclature used is the following:

$A_{h} \quad$ Hamaker constant,

$A^{\prime} \quad$ amplitude of the small perturbation of the solid film,

$D \quad$ flexural rigidity of the solid film,

$e \quad$ charge of electron,

$E \quad$ Young's modulus,

$f \quad$ sum of the surface stresses at the top and bottom surfaces of the solid film,

$F \quad$ resultant membrane force of the solid film,

$h$ thickness of the solid film,

$h_{c} \quad$ critical thickness of the solid film,

$h^{\prime} \quad$ perturbated thickness of solid film,

$H$ thickness of the liquid layer,

$H_{c} \quad$ critical thickness of the liquid layer,

$k_{B} \quad$ Boltzmann constant,

$L \quad$ wavelength,

$L_{c r} \quad$ critical wavelength,

$n$ number density,

$T$ absolute temperature,

$u \quad$ in-plane displacement in the $x$ direction,

$v \quad$ in-plane displacement in the $y$ direction,

$w \quad$ free energy density,

$w_{\text {ESE }} \quad$ elastic strain energy density,

$w_{\mathrm{EDL}}$ electric double-layer energy density, $w_{\text {Hyd }} \quad$ hydration energy density,

$W_{F} \quad$ elastic energy within the solid film,

$W_{S} \quad$ surface component of the free energy in the SOL structure,

$w_{V} \quad$ density of volumetric component of the free energy in the SOL structure,

$W_{V} \quad$ volumetric component of the free energy in the SOL structure,

$w_{\text {vdW }} \quad$ van der Waals energy density,

$z \quad$ valence of species ions,

$\beta \quad \tanh \left(z e \phi_{s} / 4 \kappa_{B} T\right)$,

$\varepsilon \quad$ dielectric permittivity of the solvent,

$\varepsilon_{0} \quad$ strain of the first monolayer,

$\kappa \quad$ reciprocal of the Debye length,

$\lambda$ decay length,

$\mu \quad$ Poisson's radio,

$\sigma \quad$ residual stress of the solid film,

$\phi_{s} \quad$ surface potential.

The abbreviations are as follows:

DLVO vdW+EDL

ESE elastic strain energy,

Hyd hydration force,

vdW van der Waals,

$\operatorname{XDLVO}(3) \mathrm{vdW}+\mathrm{EDL}+\mathrm{hyd}$,

XDLVO(4) vdW + EDL+ hyd + ESE.

${ }^{1}$ C.M. Ho and Y.C. Tai, Annu. Rev. Fluid Mech. 30, 579 (1998).

${ }^{2}$ Z. Suo, Int. J. Solids Struct. 37, 359 (2000).

${ }^{3}$ B.N.J. Persson, J. Chem. Phys. 118, 7614 (2003).

${ }^{4}$ B.V. Derjaguin and L.D. Landau, Acta Physicochim. URSS 14, 633 (1941).

${ }^{5}$ J.W. Verwey and J.Th.G. Overbeek, Theory of the Stability of Lyophobic Colloids (Elservier, Amsterdam, 1948) (reprinted by Dover, New York, 1999).

${ }^{6}$ T. Sato and R. Ruch, Stabilization of Colloidal Dispersions by Polymer Adsorption (Dekker, New York, 1980).

${ }^{7}$ W.B. Russel, D.A. Saville, and W.R. Schowalter, Colloidal Dispersions (Cambridge University Press, Cambridge, England, 1989).

${ }^{8}$ J.N. Israelachvili, Intermolecular and Surface Forces, 2nd ed. (Academic, San Diego, CA, 1992).

${ }^{9}$ A. Majumdar and I. Mezic, J. Heat Transfer 121, 964 (1999).

${ }^{10}$ A. Majumdar and I. Mezic, Microscale Thermophys. Eng. 2, 203 (1998).

${ }^{11}$ J.W. Cahn, Acta Metall. 28, 1333 (1980).

${ }^{12}$ J.R. Rice and T.J. Chuang, J. Am. Ceram. Soc. 64, 46 (1981).

${ }^{13}$ F. Spaepen, J. Mech. Phys. Solids 44, 675 (1996).

${ }^{14}$ C.H. Wu, J. Mech. Phys. Solids 44, 2059 (1996).

${ }^{15}$ L.B. Freund, J. Mech. Phys. Solids 46, 1835 (1998).

${ }^{16}$ R.C. Cammarata, Prog. Surf. Sci. 46, 1 (1994).

${ }^{17}$ R.C. Cammarata and K. Sieradzki, Annu. Rev. Mater. Sci. 24, 215 (1994).

${ }^{18}$ H. Ibach, Surf. Sci. Rep. 29, 193 (1997).

${ }^{19}$ R. Huang and Z. Suo, Thin Solid Films 429, 273 (2003).

${ }^{20}$ J. Lyklema, in Molecular Forces, edited by B. Chu, Pontif. Acad. Sci. Scripta Varia, Vol. 31 (North-Holland, Amsterdam, 1967), pp. 181, 221.

${ }^{21}$ D.H. Napper and R.J. Hunter, in Hydrosols, edited by M. Kerker, MTP Int. Rev. Sci., Surface Chem and Colloids, series 1, 1972, pp. 7, 241; series 2, 1975, pp. 7, 161 (Butterworth, London, 1972 and 1975).

${ }^{22}$ J.A. Molina-Bolý'var, F. Galisteo-Gonza'lez, and R. Hidalgo-Alvarez, Colloids Surf., B 14, 3 (1999).

${ }^{23}$ V.A. Parsegian, R.P. Rand, and D.C. Rau, Chem. Scr. 25, 28 (1985),

${ }^{24}$ C.J. van Oss, Interfacial Forces in Aqueous Media (Dekker, New York, 1994).

${ }^{25}$ D. Grasso, K. Subramaniam, M. Butkus, K. Strevett, and J. Bergendahl, Rev. Environ. Sci. Biotech. 1, 17 (2002).

${ }^{26}$ J. Skvarla, Adv. Colloid Interface Sci. 91, 335 (2001).

${ }^{27}$ A.T. Poortinga, R. Bos, W. Norde, and H.J. Busscher, Surf. Sci. Rep. 47, 1 (2002). 
${ }^{28}$ K.D. Hobart, F.J. Kub, M. Fatemi, M.E. Twigg, P.E. Thompson, T.S. Kuan, and C.K. Inoki, J. Electron. Mater. 29, 897 (2000).

${ }^{29}$ H. Yin, R. Huang, K.D. Hobart, Z. Suo, T.S. Kuan, C.K. Inoki, S.R. Shieh, T.S. Duffy, F.J. Kub, and J.C. Sturm, J. Appl. Phys. 91, 9716 (2002).

${ }^{30} \mathrm{~S}$. Timoshenko and S. Woinowsk-Krieger, Theory of Plates and Shells, 2nd ed. (McGraw-Hill, New York, 1987).

${ }^{31}$ Z. Suo and Z.Y. Zhang, Phys. Rev. B 58, 5116 (1998).

${ }^{32}$ Y.P. Zhao, Arch. Appl. Mech. 72, 77 (2002).
${ }^{33}$ M. Gavish, R. Popovitz-Biro, M. Lahav, and L. Leiserowitz, Science 250, 973 (1990).

${ }^{34}$ J. Majewski, R. Popovitz-Biro, K. Kjaer, J. Als-Neilsen, M. Lahav, and L. Leiserowitz, J. Phys. Chem. 98, 4087 (1994).

${ }^{35}$ P. Popovitz-Biro, J.L. Wang, J. Majewski, E. Shavit, L. Leiserowitz, and M. Lahav, J. Am. Chem. Soc. 116, 1179 (1994).

${ }^{36}$ W.J. Huisman, J.F. Peters, M.J. Zwanenburg, S.A. deVries, T.E. Derry, D. Abernathy, and J.F. van derVeen, Nature (London) 390, 379 (1997). 